\title{
Simulation modelling of the effect of a random disturbance on biodiversity of a mathematical model of mutualism between two interacting yeast species
}

\author{
Eke, Nwagrabe ${ }^{1}$; Atsu, J. U. ${ }^{2}$; Ekaka-a, E. $\mathrm{N}^{3}$ \\ ${ }^{1}$ Department of Mathematics/Statistics, Ignatius Ajuru University of Education, Port Harcourt, Rivers State \\ ${ }^{2}$ Department of Mathematics/Statistics, Cross River University of Technology, Calabar, Nigeria. \\ ${ }^{3}$ Department of Mathematics, Rivers State University Nkporlu-Oroworukwo, Port Harcourt, Rivers State
}

\begin{abstract}
The effect of a random disturbance on the ecosystem is one of the oldest scientific observations of which its effect on biodiversity is no exception. We have used ODE 45 numerical scheme to tackle this problem. The novel results that we have obtained have not been seen elsewhere; these are presented and fully discussed quantitatively.
\end{abstract}

Keywords - Random disturbance, numerical scheme, biodiversity, dynamical system, stochastic, deterministic dynamical system.

\section{INTRODUCTION}

An ecological dynamical system is inherently stochastic in its scientific construction and definition. In this scenario, a deterministic definition of an ecological dynamical system is a special case of a stochastic ecological system that is more highly vulnerable to random disturbance which can be attributed to the other environmental and climatic factors and other characteristics of the ecosystem which we cannot go into in detailed discussion. However, there are two factors that may have a high potential to influence the performance of biodiversity gain. One of these factors could be a conducive steady environment that is less hostile to interaction between yeast populations. The other factor could be attributed to an ecological system where human activities do not have a huge impact on the growing yeast species. These two factors put together are capable to improve the performance of yeast species in terms of their yields that can mimic strong evidence of biodiversity gain. In other words, a random noise disturbance in terms of these mentioned factors may not necessarily bring about biodiversity loss but are capable to increase the magnitude of biodiversity gain.

\section{MATERIALS AND METHODS}

We have considered a semi - stochastic fashion of our deterministic dynamical system in which a dynamical system with two random noise perturbation scenarios of 0.01 and 0.1 in the first instance and next for a random noise perturbation of 0.8 . This method is based on the 150 percent variation of the inter-competition coefficients together.

\section{RESULTS}

The corresponding results of this study are presented in Table 1, Table 2, Table 3, Table 4, Table 5, and Table 6

Table.1: Quantifying the effect of a random disturbance having the intensity of 0.01 on biodiversity gain using ODE 45 numerical scheme. Scenario One

\begin{tabular}{|c|c|c|c|c|c|c|}
\hline Example & $x(t)$ & $x_{m}(t)$ & $B G(\%)$ & $y(t)$ & $y_{m}(t)$ & $B G(\%)$ \\
\hline 1 & 4.0000 & 4.0000 & 0 & 10.0000 & 10.0000 & 0 \\
\hline 2 & 4.4497 & 4.5132 & 1.4276 & 10.7618 & 10.8509 & 0.8273 \\
\hline 3 & 4.9514 & 5.1133 & 3.2706 & 11.5776 & 11.7320 & 1.3340 \\
\hline 4 & 5.5111 & 5.7691 & 4.6831 & 12.4505 & 12.6952 & 1.9650 \\
\hline 5 & 6.1356 & 6.5317 & 6.4556 & 13.3844 & 13.7425 & 2.6755 \\
\hline 6 & 6.8325 & 7.3519 & 7.6024 & 14.3829 & 14.8480 & 3.2337 \\
\hline 7 & 7.6102 & 8.2747 & 8.7320 & 15.4502 & 16.0596 & 3.9444 \\
\hline 8 & 8.4778 & 9.3598 & 10.4040 & 16.5906 & 17.3229 & 4.4139 \\
\hline
\end{tabular}




\begin{tabular}{|c|c|c|c|c|c|c|}
\hline Example & $x(t)$ & $x_{m}(t)$ & $B G(\%)$ & $y(t)$ & $y_{m}(t)$ & $B G(\%)$ \\
\hline 9 & 9.4456 & 10.6178 & 12.4105 & 17.8090 & 18.7357 & 5.2036 \\
\hline 10 & 10.5247 & 12.0170 & 14.1789 & 19.1103 & 20.2260 & 5.8379 \\
\hline 11 & 11.7273 & 13.5941 & 15.9187 & 20.5002 & 21.8492 & 6.5805 \\
\hline 12 & 13.0666 & 15.3911 & 17.7895 & 21.9847 & 23.6066 & 7.3774 \\
\hline 13 & 14.5569 & 17.4257 & 19.7075 & 23.5705 & 25.5991 & 8.6065 \\
\hline 14 & 16.2134 & 19.7543 & 21.8394 & 25.2650 & 27.7541 & 9.8517 \\
\hline 15 & 18.0522 & 22.4048 & 24.1112 & 27.0765 & 30.0533 & 10.9939 \\
\hline 16 & 20.0902 & 25.4003 & 26.4313 & 29.0141 & 32.5986 & 12.3545 \\
\hline 17 & 22.3450 & 28.8608 & 29.1598 & 31.0880 & 35.4470 & 14.0217 \\
\hline 18 & 24.8344 & 32.6905 & 31.6343 & 33.3096 & 38.6581 & 16.0567 \\
\hline 19 & 27.5763 & 37.0962 & 34.5221 & 35.6920 & 42.2245 & 18.3025 \\
\hline 20 & 30.5884 & 42.0707 & 37.5382 & 38.2492 & 46.2160 & 20.8286 \\
\hline
\end{tabular}

Table.2: Quantifying the effect of a random disturbance having the intensity of 0.01 on biodiversity gain using ODE 45 numerical scheme. Scenario Two

\begin{tabular}{|c|c|c|c|c|c|c|}
\hline Example & $x(t)$ & $x_{m}(t)$ & $B G(\%)$ & $y(t)$ & $y_{m}(t)$ & $B G(\%)$ \\
\hline 1 & 4.0000 & 4.0000 & 0 & 10.0000 & 10.0000 & 0 \\
\hline 2 & 4.4497 & 4.5237 & 1.6635 & 10.7618 & 10.8162 & 0.5054 \\
\hline 3 & 4.9514 & 5.1350 & 3.7100 & 11.5776 & 11.7026 & 1.0797 \\
\hline 4 & 5.5111 & 5.8552 & 6.2449 & 12.4505 & 12.7082 & 2.0699 \\
\hline 5 & 6.1356 & 6.6134 & 7.7883 & 13.3844 & 13.7418 & 2.6703 \\
\hline 6 & 6.8325 & 7.5144 & 9.9797 & 14.3829 & 14.9599 & 4.0116 \\
\hline 7 & 7.6102 & 8.4799 & 11.4283 & 15.4502 & 16.2132 & 4.9386 \\
\hline 8 & 8.4778 & 9.5569 & 12.7289 & 16.5906 & 17.5598 & 5.8418 \\
\hline 9 & 9.4456 & 10.8277 & 14.6327 & 17.8090 & 19.0138 & 6.7651 \\
\hline 10 & 10.5247 & 12.2314 & 16.2166 & 19.1103 & 20.5377 & 7.4689 \\
\hline 11 & 11.7273 & 13.8468 & 18.0734 & 20.5002 & 22.2450 & 8.5111 \\
\hline 12 & 13.0666 & 15.6921 & 20.0927 & 21.9847 & 24.0618 & 9.4480 \\
\hline 13 & 14.5569 & 17.7736 & 22.0973 & 23.5705 & 26.0330 & 10.4475 \\
\hline 14 & 16.2134 & 20.1278 & 24.1427 & 25.2650 & 28.1513 & 11.4237 \\
\hline 15 & 18.0522 & 22.7965 & 26.2811 & 27.0765 & 30.5581 & 12.8584 \\
\hline 16 & 20.0902 & 25.8497 & 28.6678 & 29.0141 & 33.2120 & 14.4686 \\
\hline 17 & 22.3450 & 29.3634 & 31.4092 & 31.0880 & 36.0546 & 15.9761 \\
\hline 18 & 24.8344 & 33.3098 & 34.1278 & 33.3096 & 39.2617 & 17.8690 \\
\hline 19 & 27.5763 & 37.7921 & 37.0457 & 35.6920 & 42.9179 & 20.2453 \\
\hline 20 & 30.5884 & 42.8728 & 40.1607 & 38.2492 & 46.9771 & 22.8184 \\
\hline
\end{tabular}

Table.3: Quantifying the effect of a random disturbance having the intensity of 0.1 on biodiversity gain using ODE 45 numerical scheme. Scenario Three

\begin{tabular}{|c|c|c|c|c|c|c|}
\hline Example & $x(t)$ & $x_{m}(t)$ & $B G(\%)$ & $y(t)$ & $y_{m}(t)$ & $B G(\%)$ \\
\hline 1 & 4.0000 & 4.0000 & 0 & 10.0000 & 10.0000 & 0 \\
\hline 2 & 4.4497 & 4.5334 & 1.8803 & 10.7618 & 10.8330 & 0.6613 \\
\hline 3 & 4.9514 & 5.0793 & 2.5839 & 11.5776 & 11.7789 & 1.7395 \\
\hline 4 & 5.5111 & 5.7124 & 3.6543 & 12.4505 & 12.7405 & 2.3287 \\
\hline 5 & 6.1356 & 6.4569 & 5.2377 & 13.3844 & 13.7864 & 3.0037 \\
\hline 6 & 6.8325 & 7.2961 & 6.7848 & 14.3829 & 14.9548 & 3.9766 \\
\hline 7 & 7.6102 & 8.2988 & 9.0489 & 15.4502 & 16.1720 & 4.6722 \\
\hline 8 & 8.4778 & 9.3920 & 10.7836 & 16.5906 & 17.4731 & 5.3191 \\
\hline 9 & 9.4456 & 10.6431 & 12.6781 & 17.8090 & 18.9168 & 6.2202 \\
\hline 10 & 10.5247 & 12.0703 & 14.6858 & 19.1103 & 20.4365 & 6.9397 \\
\hline
\end{tabular}




\begin{tabular}{|c|c|c|c|c|c|c|}
\hline Example & $x(t)$ & $x_{m}(t)$ & $B G(\%)$ & $y(t)$ & $y_{m}(t)$ & $B G(\%)$ \\
\hline 11 & 11.7273 & 13.6683 & 16.5511 & 20.5002 & 22.1251 & 7.9260 \\
\hline 12 & 13.0666 & 15.4880 & 18.5308 & 21.9847 & 23.9678 & 9.0203 \\
\hline 13 & 14.5569 & 17.4978 & 20.2024 & 23.5705 & 25.9385 & 10.0464 \\
\hline 14 & 16.2134 & 19.8987 & 22.7301 & 25.2650 & 28.1712 & 11.5028 \\
\hline 15 & 18.0522 & 22.5636 & 24.9908 & 27.0765 & 30.5360 & 12.7766 \\
\hline 16 & 20.0902 & 25.5495 & 27.1740 & 29.0141 & 33.1085 & 14.1121 \\
\hline 17 & 22.3450 & 28.9518 & 29.5672 & 31.0880 & 36.0206 & 15.8668 \\
\hline 18 & 24.8344 & 32.8223 & 32.1648 & 33.3096 & 39.2187 & 17.7398 \\
\hline 19 & 27.5763 & 37.2030 & 34.9093 & 35.6920 & 42.7775 & 19.8519 \\
\hline 20 & 30.5884 & 42.1760 & 37.8825 & 38.2492 & 46.7649 & 22.2637 \\
\hline
\end{tabular}

Table.4: Quantifying the effect of a random disturbance having the intensity of 0.1 on biodiversity gain using ODE 45 numerical scheme. Scenario Four

\begin{tabular}{|c|c|c|c|c|c|c|}
\hline Example & $x(t)$ & $x_{m}(t)$ & $B G(\%)$ & $y(t)$ & $y_{m}(t)$ & $B G(\%)$ \\
\hline 1 & 4.0000 & 4.0000 & 0 & 10.0000 & 10.0000 & 0 \\
\hline 2 & 4.4497 & 4.4867 & 0.8312 & 10.7618 & 10.8039 & 0.3907 \\
\hline 3 & 4.9514 & 5.0647 & 2.2898 & 11.5776 & 11.6835 & 0.9150 \\
\hline 4 & 5.5111 & 5.6951 & 3.3405 & 12.4505 & 12.6824 & 1.8624 \\
\hline 5 & 6.1356 & 6.5067 & 6.0488 & 13.3844 & 13.7450 & 2.6946 \\
\hline 6 & 6.8325 & 7.3949 & 8.2316 & 14.3829 & 14.8654 & 3.3550 \\
\hline 7 & 7.6102 & 8.3792 & 10.1059 & 15.4502 & 16.0553 & 3.9163 \\
\hline 8 & 8.4778 & 9.4514 & 11.4848 & 16.5906 & 17.3531 & 4.5954 \\
\hline 9 & 9.4456 & 10.7237 & 13.5309 & 17.8090 & 18.7569 & 5.3224 \\
\hline 10 & 10.5247 & 12.1772 & 15.7008 & 19.1103 & 20.2479 & 5.9526 \\
\hline 11 & 11.7273 & 13.7391 & 17.1551 & 20.5002 & 21.9514 & 7.0789 \\
\hline 12 & 13.0666 & 15.5557 & 19.0486 & 21.9847 & 23.7044 & 7.8224 \\
\hline 13 & 14.5569 & 17.6162 & 21.0155 & 23.5705 & 25.6845 & 8.9686 \\
\hline 14 & 16.2134 & 19.9303 & 22.9250 & 25.2650 & 27.9186 & 10.5029 \\
\hline 15 & 18.0522 & 22.5407 & 24.8641 & 27.0765 & 30.2741 & 11.8094 \\
\hline 16 & 20.0902 & 25.5231 & 27.0421 & 29.0141 & 32.9389 & 13.5275 \\
\hline 17 & 22.3450 & 28.9112 & 29.3857 & 31.0880 & 35.7840 & 15.1058 \\
\hline 18 & 24.8344 & 32.8304 & 32.1974 & 33.3096 & 38.9832 & 17.0329 \\
\hline 19 & 27.5763 & 37.2158 & 34.9561 & 35.6920 & 42.5658 & 19.2588 \\
\hline 20 & 30.5884 & 42.1994 & 37.9592 & 38.2492 & 46.5714 & 21.7578 \\
\hline
\end{tabular}

Table.5: Quantifying the effect of a random disturbance having the intensity of 0.8 on biodiversity gain using ODE 45 numerical scheme. Scenario Five

\begin{tabular}{|c|c|c|c|c|c|c|}
\hline Example & $x(t)$ & $x_{m}(t)$ & $B G(\%)$ & $y(t)$ & $y_{m}(t)$ & $B G(\%)$ \\
\hline 1 & 4.0000 & 4.0000 & 0 & 10.0000 & 10.0000 & 0 \\
\hline 2 & 4.4497 & 4.8324 & 8.6014 & 10.7618 & 11.4308 & 6.2161 \\
\hline 3 & 4.9514 & 5.8134 & 17.4109 & 11.5776 & 12.7359 & 10.0048 \\
\hline 4 & 5.5111 & 6.9156 & 25.4863 & 12.4505 & 14.2481 & 14.4378 \\
\hline 5 & 6.1356 & 8.2866 & 35.0587 & 13.3844 & 15.5423 & 16.1229 \\
\hline 6 & 6.8325 & 9.6980 & 41.9400 & 14.3829 & 17.1956 & 19.5561 \\
\hline 7 & 7.6102 & 11.2228 & 47.4713 & 15.4502 & 18.8135 & 21.7691 \\
\hline 8 & 8.4778 & 12.9207 & 52.4067 & 16.5906 & 20.7384 & 25.0008 \\
\hline 9 & 9.4456 & 15.1202 & 60.0765 & 17.8090 & 22.8115 & 28.0896 \\
\hline 10 & 10.5247 & 17.3165 & 64.5317 & 19.1103 & 25.0589 & 31.1277 \\
\hline 11 & 11.7273 & 19.9405 & 70.0344 & 20.5002 & 27.5825 & 34.5477 \\
\hline 12 & 13.0666 & 22.7270 & 73.9314 & 21.9847 & 30.4937 & 38.7040 \\
\hline
\end{tabular}




\begin{tabular}{|c|c|c|c|c|c|c|}
\hline Example & $x(t)$ & $x_{m}(t)$ & $B G(\%)$ & $y(t)$ & $y_{m}(t)$ & $B G(\%)$ \\
\hline 13 & 14.5569 & 26.0227 & 78.7651 & 23.5705 & 33.5233 & 42.2257 \\
\hline 14 & 16.2134 & 29.7098 & 83.2424 & 25.2650 & 36.7004 & 45.2614 \\
\hline 15 & 18.0522 & 34.0788 & 88.7790 & 27.0765 & 40.6045 & 49.9621 \\
\hline 16 & 20.0902 & 39.0831 & 94.5379 & 29.0141 & 44.6849 & 54.0111 \\
\hline 17 & 22.3450 & 44.9096 & 100.9828 & 31.0880 & 48.9859 & 57.5719 \\
\hline 18 & 24.8344 & 51.3077 & 106.5995 & 33.3096 & 53.9942 & 62.0979 \\
\hline 19 & 27.5763 & 58.3908 & 111.7429 & 35.6920 & 60.0885 & 68.3532 \\
\hline 20 & 30.5884 & 66.7729 & 118.2950 & 38.2492 & 66.8022 & 74.6498 \\
\hline
\end{tabular}

Table.6: Quantifying the effect of a random disturbance having the intensity of 0.8 on biodiversity gain using ODE 45 numerical scheme. Scenario Six

\begin{tabular}{|c|c|c|c|c|c|c|}
\hline Example & $x(t)$ & $x_{m}(t)$ & $B G(\%)$ & $y(t)$ & $y_{m}(t)$ & $B G(\%)$ \\
\hline 1 & 4.0000 & 4.0000 & 0 & 10.0000 & 10.0000 & 0 \\
\hline 2 & 4.4497 & 4.8883 & 9.8575 & 10.7618 & 11.1320 & 3.4394 \\
\hline 3 & 4.9514 & 5.9583 & 20.3360 & 11.5776 & 12.4481 & 7.5191 \\
\hline 4 & 5.5111 & 6.9956 & 26.9379 & 12.4505 & 13.8045 & 10.8751 \\
\hline 5 & 6.1356 & 8.2553 & 34.5478 & 13.3844 & 15.3543 & 14.7181 \\
\hline 6 & 6.8325 & 9.7534 & 42.7497 & 14.3829 & 17.0949 & 18.8558 \\
\hline 7 & 7.6102 & 11.3727 & 49.4406 & 15.4502 & 18.7917 & 21.6279 \\
\hline 8 & 8.4778 & 13.1416 & 55.0121 & 16.5906 & 20.8438 & 25.6357 \\
\hline 9 & 9.4456 & 15.2574 & 61.5298 & 17.8090 & 22.9964 & 29.1282 \\
\hline 10 & 10.5247 & 17.3844 & 65.1771 & 19.1103 & 25.2481 & 32.1174 \\
\hline 11 & 11.7273 & 19.9983 & 70.5275 & 20.5002 & 27.7474 & 35.3519 \\
\hline 12 & 13.0666 & 23.1748 & 77.3585 & 21.9847 & 30.5111 & 38.7835 \\
\hline 13 & 14.5569 & 26.8443 & 84.4089 & 23.5705 & 33.4027 & 41.7137 \\
\hline 14 & 16.2134 & 31.0127 & 91.2778 & 25.2650 & 36.8937 & 46.0266 \\
\hline 15 & 18.0522 & 35.6022 & 97.2178 & 27.0765 & 40.4674 & 49.4558 \\
\hline 16 & 20.0902 & 40.7135 & 102.6534 & 29.0141 & 44.7757 & 54.3243 \\
\hline 17 & 22.3450 & 46.7144 & 109.0600 & 31.0880 & 49.3679 & 58.8006 \\
\hline 18 & 24.8344 & 53.3229 & 114.7143 & 33.3096 & 54.9415 & 64.9419 \\
\hline 19 & 27.5763 & 61.0529 & 121.3966 & 35.6920 & 60.9044 & 70.6391 \\
\hline 20 & 30.5884 & 69.7168 & 127.9196 & 38.2492 & 67.8519 & 77.3942 \\
\hline
\end{tabular}

\section{DISCUSSION OF RESULTS}

For a random noise variation of 0.01 and 0.1 over repeated simulations as shown on Table 1 to table 4, we have observed a relatively smaller prediction of biodiversity gain whereas for a random noise variation of 0.8 , we have observed a bigger prediction of biodiversity gain. On the basis of this present analysis, a random noise inclusion which may be considered as having a negative effect, has turned out in this scenario to have a positive effect on the ecological services.

\section{CONCLUSION}

Not all random noise driven factors do predict biodiversity loss. We have utilized the technique of a numerical simulation to predict that a higher random noise perturbation has the potential to predict bigger volumes of biodiversity gain than a lower random noise perturbation, provided the two yeast species interact mutually on the simplifying assumption of varying the inter-competition coefficients together. This numerical result complements a popular ecological idea that in a harsh ecological environment, species tend to benefit each other (Ekaka-a 2009, Ford, Lumb, Ekaka-a 2010). The predictions of this present study are based on the one hundred and fifty (150) percent variations of the inter competition coefficients together on the simplifying assumption that the intra-competition coefficients outweigh the inter-competition coefficients. However, we have not extended this idea to the scenario of two (2) competing yeast species undergoing a random noise perturbation. This will be the subject of our next investigation.

\section{REFERENCES}

[1] Atsu, J. U. \& Ekaka-a, E. N. (2017). Modeling the policy implications of biodiversity loss: A case study 
of the Cross River national park, south - south Nigeria. International Journal of Pure and Applied Science, Cambridge Research and Publications. vol 10 No. 1 ; pp 30-37.

[2] Atsu, J. U. \& Ekaka-a, E. N. (2017). Quantifying the impact of changing intrinsic growth rate on the biodiversity of the forest resource biomass: implications for the Cross River State forest resource at the Cross River National Park, South - South, Nigeria: African Scholar Journal of Pure and Applied Science, 7(1); 117 - 130.

[3] De Mazancourt, C., Isbell, F., Larocque, A., Berendse, F., De Luca, E., Grace, J.B et al. (2013). Predicting ecosystem stability from community composition and biodiversity. Ecology Letters, DOI: 10.1111/ele.12088.

[4] Ernest, S.K.M. \& Brown, J.H. (2001). Homeostasis and compensation: the role of species and resources in ecosystem stability. Ecology, 82, 2118-2132.

[5] Fowler, M.S., Laakso, J., Kaitala, V., Ruokolainen, L. \& Ranta, E. (2012). Species dynamics alter community diversity-biomass stability relationships. Ecol. Lett., 15, 1387-1396.

[6] Gonzalez, A. \& Descamps-Julien, B. (2004). Population and community variability in randomly fluctuating environments. Oikos, 106, 105-116.

[7] Grman, E., Lau, J.A., Donald, R., Schoolmaster, J. \& Gross, K.L. (2010). Mechanisms contributing to stability in ecosystem function depend on the environmental context. Ecol. Lett., 13, 1400-1410.

[8] Hector, A., Hautier, Y., Saner, P., Wacker, L., Bagchi, R., Joshi, J. et al. (2010). General stabilizing effects of plant diversity on grassland productivity through population asynchrony and overyielding. Ecology, 91, 2213-2220.

[9] Loreau, M.. \& de Mazancourt, C.. (2013). Biodiversity and ecosystem stability: a synthesis of underlying mechanisms. Ecol. Lett., DOI: 10.1111/ele.12073.

[10] MacArthur, R. (1955). Fluctuations of Animal Populations, and a Measure of Community Stability. Ecology, 36, 533-536.

[11] Marquard, E., Weigelt, A., Roscher, C., Gubsch, M., Lipowsky, A. \& Schmid, B. (2009). Positive biodiversity-productivity relationship due to increased plant density. J. Ecol., 97, 696-704.

[12] May, R.M. (1973). Stability and complexity in model ecosystems. 2001, Princeton Landmarks in Biology edn. Princeton University Press, Princeton. McCann, K.S. (2000). The diversity-stability debate. Nature, 405, 228-233.
[13] McNaughton, S.J. (1977). Diversity and stability of ecological communities: a comment on the role of empiricism in ecology. Am. Nat., 111, 515-525.

[14] Mutshinda, C.M., O’Hara, R.B. \& Woiwod, I.P. (2009). What drives community dynamics? Proc. Biol. Sci., 276, 2923-2929.

[15] Proulx, R., Wirth, C., Voigt, W., Weigelt, A., Roscher, C., Attinger, S. et al.(2010). Diversity Promotes Temporal Stability across Levels of Ecosystem Organization in Experimental Grasslands. PLoS ONE, 5, e13382. 